\title{
Pédagogie numérique pour des travaux pratiques d'électronique analogique en licence « sciences pour l'ingénieur »
}

\author{
Jean-Marc Routoure ${ }^{1}$, Corentin Jorel ${ }^{1}$, Didier Robbes ${ }^{1}$, Anne Garnavault-Remy ${ }^{2}$ et Jeanine \\ Berthier $^{2}$ \\ Correspondant : jean-marc.routoure@unicaen.fr
}

${ }^{1}$ UFR de sciences, Université de Caen Normandie, Boulevard du Maréchal Juin, 14050 Caen Cedex

${ }^{2}$ Centre d'Etude Multimédia Universitaire, Université de Caen Normandie, Boulevard Yitzhak Rabin, 14123 Ifs

\section{RÉSUMÉ :}

Le succès de la plateforme youtube de partage de vidéos montre l'attrait du public et donc aussi des étudiants de nos filières scientifiques pour le support vidéo. C'est le point de départ d'un projet appelé TPELECTROSPI. Il a consisté à remplacer l'adage bien connu « un dessin vaut mieux qu'un long discours » utilisé dans les textes de TP pour expliquer les pratiques expérimentales par une version plus contemporaine " une vidéo vaut mieux qu'un long discours ». Cette évolution éditoriale a été l'occasion de remettre en cause certains fondamentaux des travaux pratiques : le texte de TP, le travail préparatoire et le compte-rendu par l'utilisation intensive d'outils de pédagogie numérique. Cette expérience a été appliquée pour un cours d'électronique analogique du second semestre de la mention « sciences pour l'ingénieur » de la licence «sciences, technologie, santé » de l'Université de Caen Normandie. Un questionnaire mis en place en fin de cours et les statistiques d'utilisation de la plateforme numérique ont permis de montrer l'intérêt du dispositif pour les étudiants. Cette expérience sera partagée avec les autres collègues du département pour une extension éventuelle à d'autres enseignements.

Mots clés : dispositif hybride, électronique analogique, travaux pratiques 2.0, pédagogique numérique.

\section{INTRODUCTION}

Dans cet article, une expérience pédagogique effectuée en seconde année de licence «sciences, technologie, santé » de la mention «Sciences pour l'ingénieur » de l'Université de Caen Normandie est présentée. L'expérience a consisté d'une part en l'utilisation de la plateforme de pédagogie numérique moodle pour vérifier les pré-requis du cours, pour préparer les séances de CM et de TD, pour le travail préparatoire de TP et les compte-rendus et d'autre part en une réécriture des textes de TP en utilisant des supports vidéo et une carte heuristique en guise de texte de TP.

Cette expérience («TPELECTROSPI ») est dans un premier temps décrite. Dans une seconde partie, les résultats d'une enquête réalisée auprès des étudiants sont présentés et analysés.

\section{PRESENTATION DE L'EXPÉRIENCE TPELECTROSPI}

\subsection{Le contexte}

Tous les ans, le CEMU (Centre d'Etude Multimédia Universitaire) de l'Université de Caen Normandie organise un appel à projet de pédagogie numérique appelé INITIATICE. C'est dans ce contexte que TPELECTROSPI a été développé. Le projet a bénéficié du support du CEMU en 
terme de pédagogie (numérique ou pas) et du soutien des équipes de tournage pour les séquences vidéos réalisées dans les salles de TP.

\subsection{Le programme du cours d'électronique analogique et ses objectifs}

Le document d'habilitation de la licence indique que le programme de cet enseignement est « Réponse d'un circuit électronique linéaire, Filtre du premier ordre et diagramme de Bode. ». Des objectifs pédagogiques suivant ont été déduits :

- Etre capable d'analyser le fonctionnement d'un circuit réalisant un filtre du premier et du second ordre

- Etre capable de mesurer les performances d'un filtre du premier et du second ordre

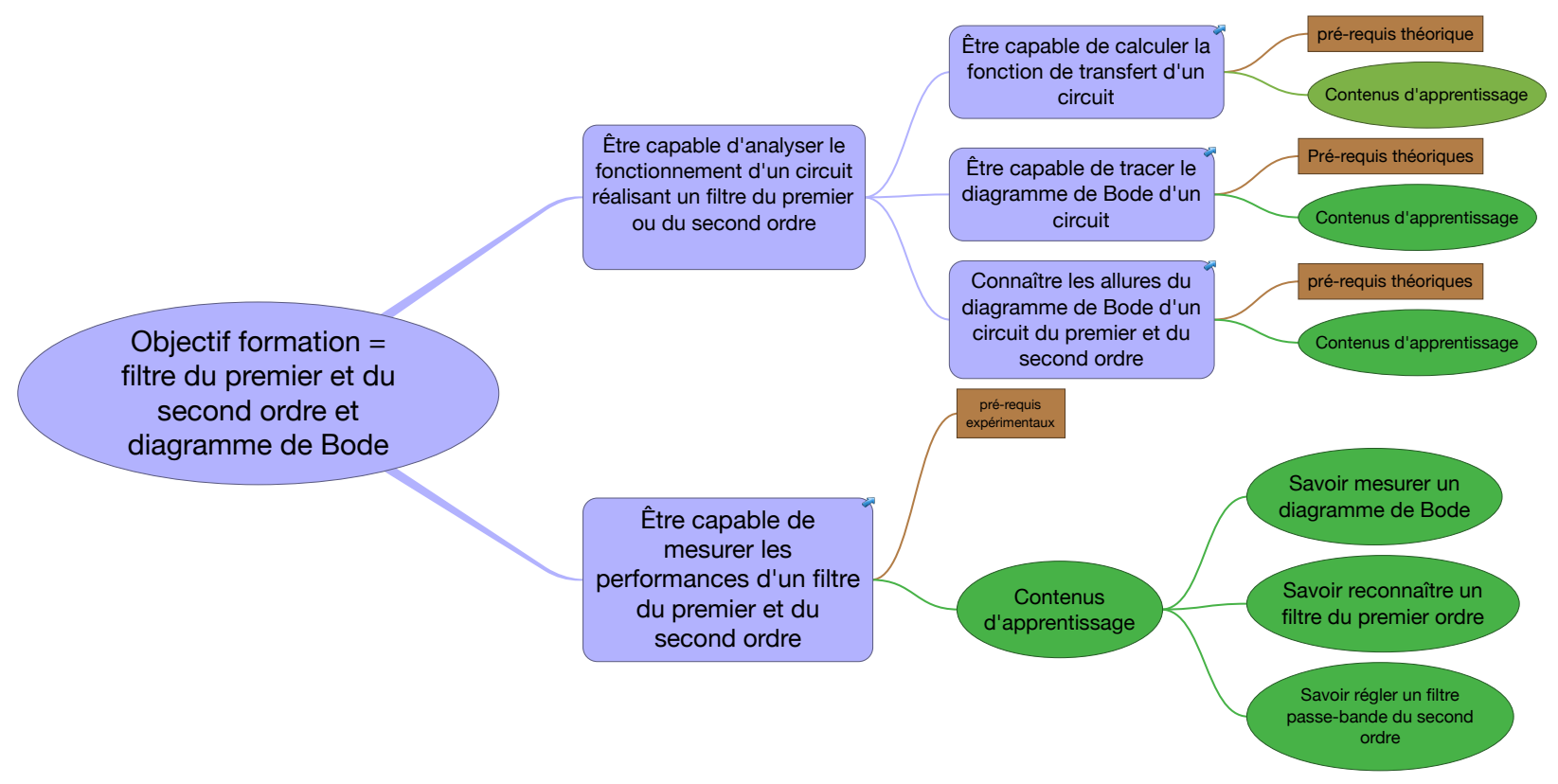

Figure 1 : table heuristique présentant les objectifs du cours

La décomposition plus fine de ces objectifs est indiquée sur la figure 1. Cette formalisation des objectifs pédagogiques a permis d'identifier des pré-requis théoriques et expérimentaux, de structurer les contenus d'apprentissage pour chaque objectif pédagogique et d'en déduire la stratégie pédagogique adaptée.

L'utilisation de la plateforme de pédagogie numérique moodle a permis de proposer un dispositif d'enseignement mixte : synchrone et asynchrone, à distance et en présentiel et avec des activités individuelles et des activités de groupe (non décrites ici).

\subsection{Les situations d'apprentissage}

Pour chaque contenu d'apprentissage associé à un objectif pédagogique, des situations pédagogiques ont pu être identifiées. Dans le cadre de ce dispositif, 4 situations d'apprentissage ont pu être définies :

- vérification des pré-requis à distance,

- cours magistral en présentiel,

- TD en présentiel,

- TP en présentiel. 
Excepté pour la vérification des pré-requis, la pédagogie numérique n'a pas modifié les pratiques pédagogiques classiquement utilisées en sciences expérimentales. L'identification des différentes situations d'apprentissages et objectifs pédagogiques permet de structurer le cours pour l'enseignant mais surtout pour les étudiants : le travail permet d'atteindre les objectifs du cours. Pour l'enseignant, chaque CM ou TD en présentiel correspond à un objectif pédagogique.

Pour chaque situation d'apprentissage, nous avons cherché à identifier les rôles, les outils, l'environnement, la durée, les instants, les ressources et enfin les modalités d'évaluation. Cela est décrit dans les paragraphes suivants.

\subsubsection{Vérification des pré-requis}

Pour être capable de calculer la fonction de transfert d'un circuit, il faut maitriser l'utilisation des nombres complexes, les échelles logarithmiques et le décibel. Ainsi sur l'espace moodle du cours, des ressources ont été mises à disposition des étudiants (un lien vers un cours en ligne [1] ainsi que deux questionnaires d'auto-évaluation disponibles dans un temps limité). Le premier questionnaire (noté PR1 dans les résultats de l'enquête auprès des étudiants) portait sur l'utilisation des nombres complexes et le second questionnaire (noté PR2) sur les échelles logarithmiques et le décibel.

Le nombre de tentatives au questionnaire était illimité avec un objectif de $90 \%$ de réussite pour valider cette auto-évaluation. Les questionnaires comprenaient des questions de calcul de valeurs numériques. A chaque nouvelle tentative, de nouvelles valeurs numériques étaient choisies.

La phase de vérification des pré-requis a été limitée aux 4 premières semaines du semestre.

\subsubsection{Cours magistraux et travaux dirigées}

Cette situation d'apprentissage a démarré après les 4 premières semaines de vérification des prérequis. Elle a consisté en une séance de CM et 1 séance de TD d'1h30 chacune pendant 5 semaines pour un total de $7 \mathrm{~h} \mathrm{CM}$ et $7 \mathrm{~h} \mathrm{TD}$ environ. Pour imposer aux étudiants une préparation des séances, la plateforme de pédagogie numérique a été utilisée.

Pour la préparation des CM, un QCM comprenant des questions de cours a été ouvert à la fin de chaque $\mathrm{CM}$ et fermé au début du CM suivant pour imposer aux étudiants une relecture de leur note manuscrite. Pour la préparation des TD, il a été demandé aux étudiants de déposer sous moodle une photographie ou un «scan » de leur note de préparation du TD. Pour ces deux actions, il faut noter qu'en dehors de la mise en place de l'infrastructure du cours (l'écriture des questions et la mise en place des zones de dépôts de fichier), ces techniques permettent d'imposer un travail personnel aux étudiants et n'entrainent aucune charge liée aux corrections.

Un devoir écrit sur table ainsi qu'un devoir à la maison ont permis l'évaluation du premier objectif du cours.

\subsubsection{Travaux pratiques}

Comme l'indique la figure 1, le cours comporte 3 contenus d'apprentissage qui ont naturellement été effectués sous la forme de 3 séances de travaux pratiques de 3 h qui se sont déroulés pendant les 3 dernières semaines du semestre un fois que les CM et TD étaient terminés. Les travaux pratiques ont été réalisés en binômes. Les sujets des 3 TPs étaient :

- Mesure du diagramme de Bode sur une boite noire (5 points de mesure par décade et 3 décades de fréquence),

- Mesure du diagramme de Bode d'un filtre d'ordre 1 entre $100 \mathrm{~Hz}$ et $1 \mathrm{MHz}$,

- Décodage d'un signal codé en morse et très fortement bruité. 
Avant chaque séance, le travail préparatoire demandé consistait en un questionnaire sous moodle permettant de vérifier les pré-requis expérimentaux et de préparer efficacement la séance. Les modalités du questionnaire étaient similaires à celles de la vérification des pré-requis. Ce questionnaire devait avoir été effectué avant le début de la séance de TP. La figure 3 illustre une des questions posées lors du travail préparatoire de la première séance de TP. Il s'agit ici de vérifier que les étudiants seront capables de mesurer correctement le déphasage de deux signaux sinusoïdaux.

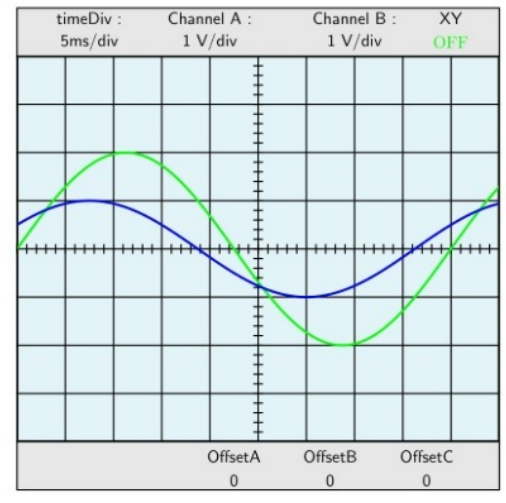

Question : dans l'oscillogramme ci-dessus, quelle est la valeur numérique du déphasage (en degrée) entre le signal représenté en bleu et le signal représenté en vert.

réponse :

Figure 2 : exemple de question sur la plateforme moodle pour le travail préparatoire demandé en TP

Une fois que le travail préparatoire était validé, les ressources et le texte de TP étaient rendus disponibles sur la plateforme moodle. Un exemple de « texte » de TP est indiqué sur la figure 3.

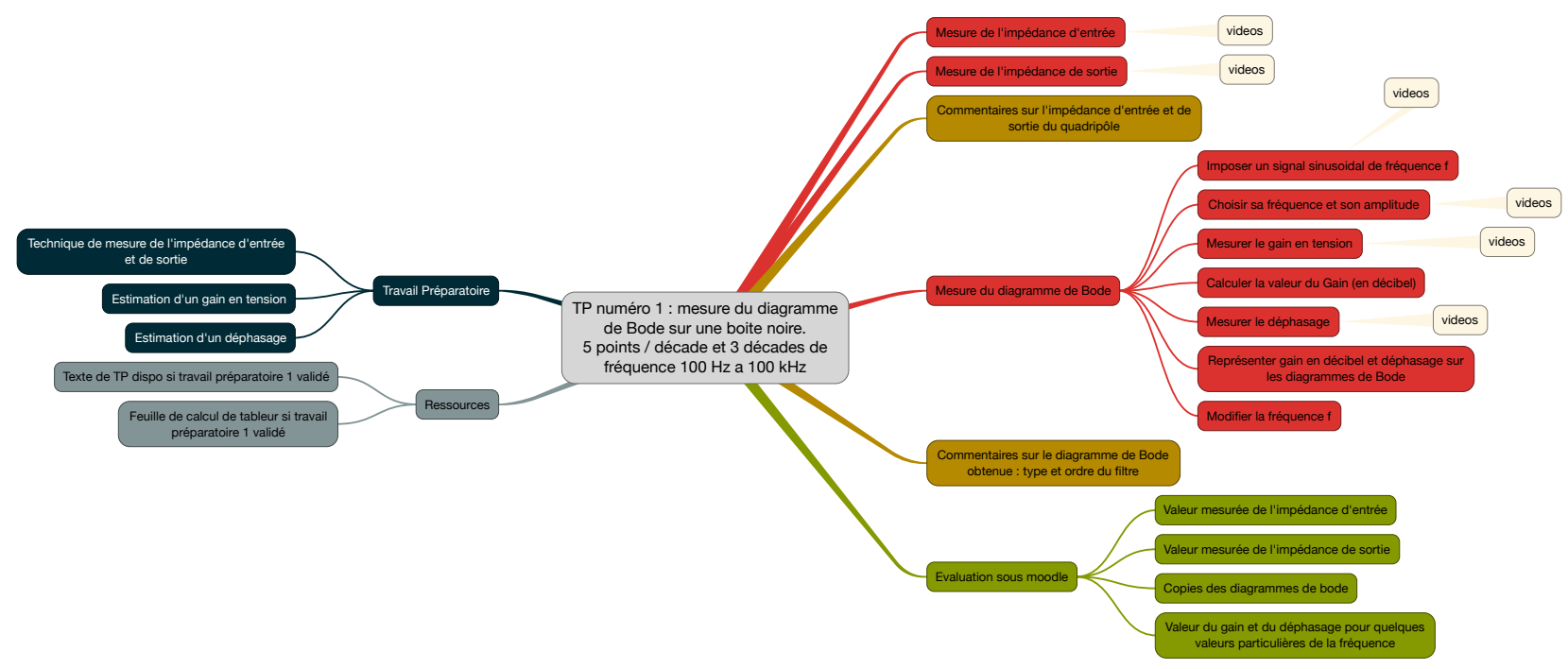

Figure 3 : texte de TP présenté sous la forme d'une table heuristique.

Le texte de TP est fourni sous forme de table heuristique. Il indique en son centre l'objectif du travail pratique. Sur la gauche est rappelé le travail préparatoire demandé et les ressources disponibles et sur la droite on trouve en rouge, les pratiques expérimentales à effectuer pendant la séance de $\mathrm{TP}$, en marron l'analyse des mesures à effectuer et en vert le compte-rendu à effectuer. 
Pour ces TP, il n'a pas été demandé de compte-rendu papier mais de remplir un questionnaire moodle et de fournir le diagramme de Bode mesuré pendant la séance. La plateforme moodle permet d'imposer un cadre strict pour les dates limites et les devoirs à remettre.

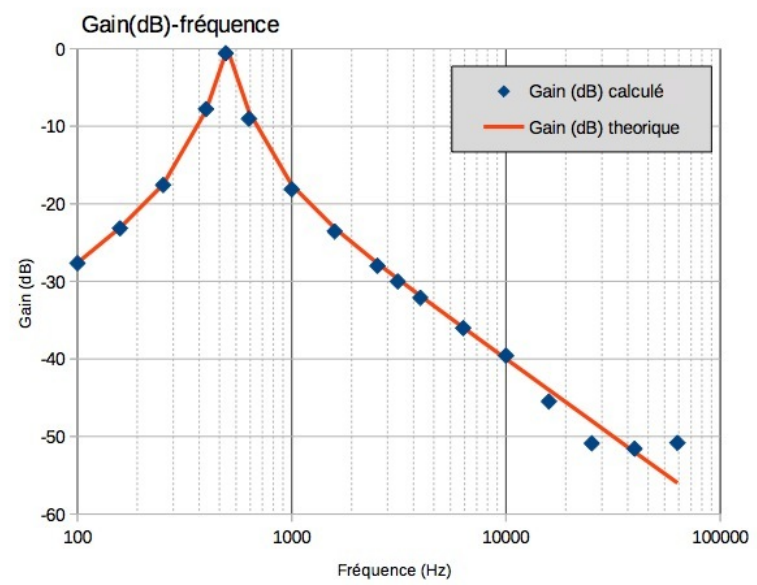

Figure 4 : exemple de production des étudiants : la ligne continue a été calculée lors du travail préparatoire et les symboles ont été ajoutés suite aux mesures.

La figure 4 donne un exemple du résultat du travail préparatoire (Gain $(\mathrm{dB})$ théorique représenté avec une ligne continue) et des mesures effectuées pendant la séance de TP (Gain (dB) calculé représenté par des losanges). Pour cette figure, le calcul du module du gain en tension théorique avait été demandé pendant le travail préparatoire.

Comme l'indique le texte de TP sur la figure 3, des séquences vidéos étaient disponibles avant et pendant la séance de TP pour guider les étudiants dans leurs pratiques expérimentales. Quelques extraits de la séquence vidéo ayant pour thème « imposer un signal sinusoidal » sont donnés sur la figure 5 . 

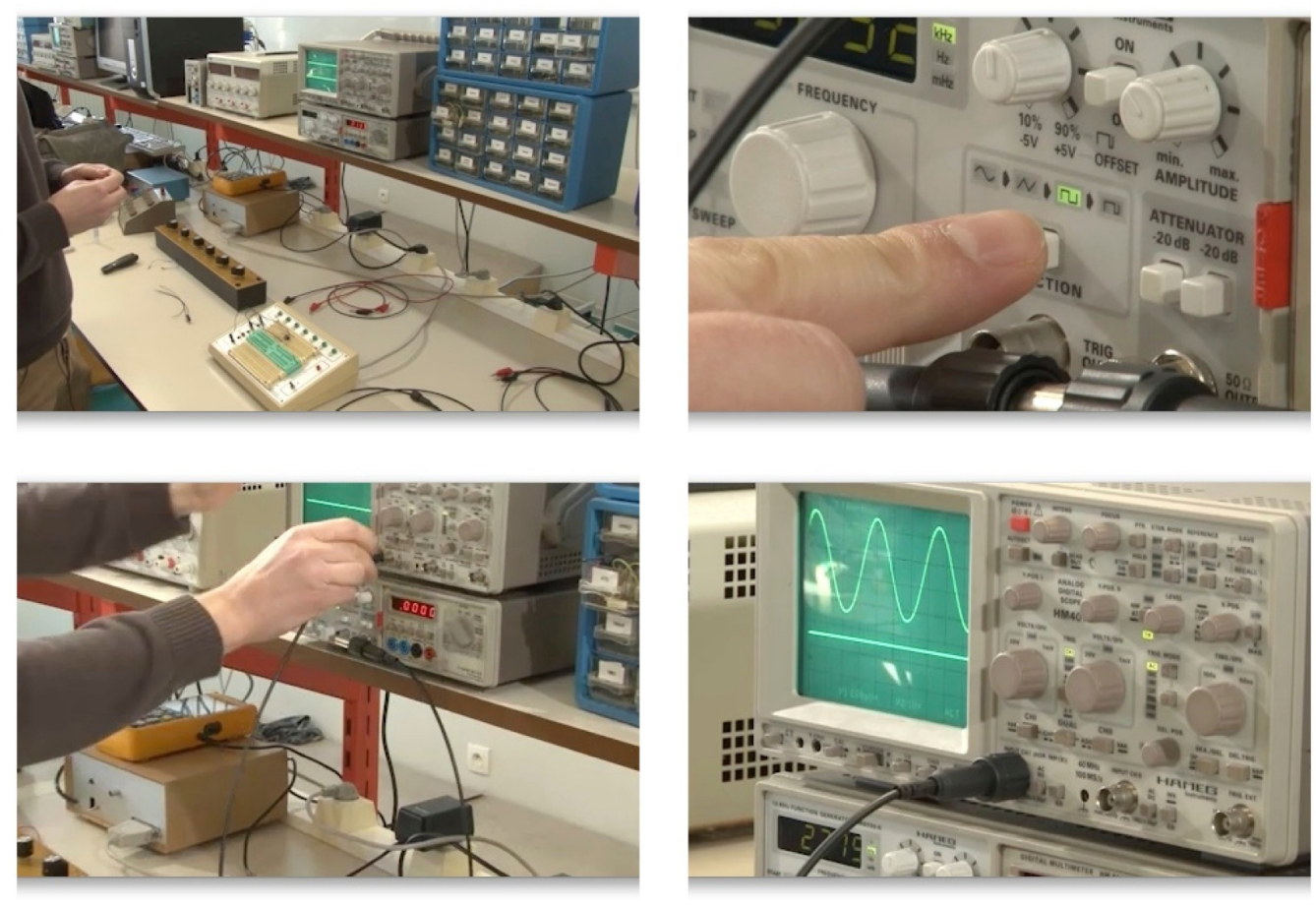

Figure 5 : extraits de la séquence vidéo : « imposer un signal sinusoïdal ».

La disponibilité de ces séquences vidéos ont permis de modifier le rôle de l'encadrant de travaux pratiques qui devient une ressource support pour les étudiants. Le rôle et le comportement des étudiants sont également modifiés. Généralement passif lors des explications techniques données par l'encadrant de TP, les étudiants ont pu revoir plusieurs fois la même séquence et la mettre en pause pour s'assurer que les manipulations réalisées étaient identiques à celles visionnées dans la séquence vidéo.

\section{RÉSULTATS DE L'ENQUETE EFFECTUÉE AUPRES DES ÉTUDIANTS}

Pour évaluer l'impact de ce projet sur les étudiants de la seconde année de licence «sciences pour l'ingénieur », un questionnaire (basé sur les travaux proposés par [2] et [3]) a été proposé. Le questionnaire a porté sur les deux situations d'apprentissage les plus innovantes à savoir la vérification des pré-requis et les travaux pratiques et sur l'utilisation de la plateforme moodle. Les résultats complets de l'enquête sont donnés en annexe. Les étudiants devaient noter chaque proposition de 1 à 4 . La note 1 correspondant à un avis très négatif, 2 un avis négatif, 3 un avis positif et 4 un avis très positif. Ne sont reportés ici que les propositions ayant obtenues un résultat moyen supérieur ou égal à 3 .

\subsection{Vérification des pré-requis}

L'enquête indique que :

- les objectifs et les consignes des questionnaires étaient clairement définis et suffisants,

- les ressources proposées en auto-apprentissage étaient adaptées,

- l'évaluation était pertinente et qu'elle permettait de vérifier ses acquis. 
Les résultats sont sensiblement plus faibles pour le second questionnaire (PR2) que pour le premier (PR1).

En moyenne, les étudiants ont passés $2 \mathrm{~h}$ pour chaque questionnaire à étudier les ressources proposées et à répondre aux questions du QCM. Les étudiants ont effectués en moyenne 6 tentatives pour le premier questionnaire et 5 tentatives pour le second. Au moins la moitié des étudiants a répondu correctement à toutes les questions (la consultation des statistiques d'utilisation de la plateforme montre que certains étudiants ont refait plusieurs fois le questionnaire pour avoir toutes les bonnes réponses alors qu'ils avaient déjà obtenu $90 \%$ de bonnes réponses).

Les commentaires libres sont un peu plus négatifs pour le second questionnaire que pour le premier; on notera toutefois ce commentaire : « la vérification pré-requis $1 \mathrm{~m}$ 'a permis d'apprendre et de comprendre à mon rythme ».

\subsection{Travaux pratiques}

L'enquête indique que les étudiants ont trouvés que l'objectif et les consignes étaient clairement définis et suffisants, que l'accès aux ressources, contenus et activités en ligne était adapté à la réalisation du TP et que l'espace de cours était convivial et facile à utiliser. Les étudiants ont été questionnés sur les éléments qui ont facilité la réalisation du TP et le résultat de l'enquête indique :

- l'énoncé du TP sous forme de Mindmap,

- la saisie des résultats en ligne,

- les vidéos des pratiques expérimentales (75\% d'avis très favorables !).

Tous les étudiants ont consultés les vidéos et ils sont $62 \%$ à indiquer ne pas avoir rencontré de difficultés pour la réalisation du TP.

\subsection{Utilisation de la plateforme moodle pour les TP}

L'enquête a porté sur la comparaison avec un TP sans usage de la plateforme et des ressources en ligne. Cela a permis aux étudiants :

- d'être plus motivés,

- de rendre les tâches proposées plus intéressantes,

- d'être plus impliqués dans le TP,

- de faciliter l'apprentissage,

- de réfléchir sur leur manière d'apprendre,

- de gérer eux-même leurs ressources d'apprentissage,

- d'améliorer la forme des productions,

- d'améliorer la qualité des productions,

- de favoriser l'interaction entre eux.

Un des commentaires libres mérite d'être indiquer ici car il valide et conforte l'expérience : « la formation en ligne est très agréable car on peut bien organiser son travail et mieux le comprendre ».

\subsection{Commentaires}

Plus de 50\% des étudiants ont passé plus de $2 \mathrm{~h}$ sur les tests en auto-apprentissage et ont effectué en moyenne 5 fois chaque questionnaire : ce résultat montre que les outils de pédagogie numérique 
permettent de faire travailler efficacement les étudiants en autonomie. La remarque « la vérification pré-requis $1 \mathrm{~m}$ 'a permis d'apprendre et de comprendre à mon rythme » montre que certains étudiants se sont accaparés et ont appréciés cette étape du dispositif.

Aucune des nouveautés introduites en TP (cf. 3.2.2)-mindmap, travail préparatoire sous forme de QCM, saisie des comptes-rendus en ligne et vidéos n'a perturbée les étudiants. On peut même relever que $75 \%$ des étudiants ont apprécié l'utilisation des vidéos.

On peut noter que pour l'ensemble des questions posées concernant l'utilisation de la plateforme numérique, toutes les propositions proposées (voir en annexe) ont été retenues en comparaison avec des travaux pratiques classiques. La dernière proposition indique que cela ne leur a pas demandé un travail supplémentaire ! Comme déjà mentionné, on notera enfin le commentaire « La formation en ligne est très agréable car on peut bien organiser notre travail et mieux le comprendre »d'un étudiant. Tout cela montre bien l'intérêt que peut avoir l'utilisation des plateformes en ligne pour les étudiants et leur appétence pour ces nouvelles pratiques.

Les statistiques d'utilisation de la plateforme moodle sont également intéressantes : en moyenne chaque étudiant a effectué 265 actions sur la plateforme avec un minimum de 149 actions et un maximum de 358. Pour certains jours, plus de 30 actions étaient réalisées sur la plateforme.

Enfin, cette expérience n'a pas montré d'évolution des résultats obtenus par les étudiants. La note moyenne de l'élément l'année de l'expérience est de 11,7. Elle était respectivement égale à 11,28 et de 11,6 l'année précédente et 2 années précédentes.

\section{CONCLUSION}

Cet article a présenté une expérience pédagogique qui a principalement consistée à remplacer les textes de travaux pratiques par une feuille sous forme de table heuristique et à décrire les manipulations à effectuer en utilisant des vidéos. Dans cette expérience, la plateforme de pédagogie moodle a été utilisée, en support pour les vidéos mais aussi pour proposer une situation d'apprentissage pour la vérification des pré-requis, pour proposer des questionnaires permettant d'obtenir un travail personnel important et enfin pour proposer un espace pour le compte-rendu des travaux pratiques.

Une évaluation du cours a permis de montrer que les étudiants n'ont pas été perturbés dans leur apprentissage et qu'ils ont bien apprécié les séquences vidéos.

Cette expérimentation sera présentée aux collègues de l'UFR des sciences de l'Université de Caen Normandie. Une extension de cette expérimentation en vue d'enseignement à distance est également envisagée : des séances de travaux pratiques mêlant étudiants présents et étudiants connectés et utilisant le même matériel à base d'instrumentation connectée à un ordinateur semblent être une perspective plausible. Enfin, on peut citer l'article de l'arrêté du 22 janvier 2014 fixant le cadre national des formations conduisant à la délivrance des diplômes nationaux de licence, de licence professionnelle et de master : «l'usage du numérique doit permettre une pédagogie active, réactive et interactive entre étudiants et entre étudiants et équipes pédagogiques. La formation, ou une partie de celle-ci, peut être proposée selon des dispositifs hybrides par l'alternance d'activités pédagogiques en présentiel et à distance ou totalement à distance, en fonction du public concerné. ». Cette expérience s'inscrit donc complètement dans le cadre proposé par cet article et pourra inspirer les collègues d'autres universités. 


\section{Remerciements}

Les auteurs tiennent à remercier Olivier Cosme et Corinne Mouchel pour le tournage, la réalisation et le montage des séquences vidéos.

\section{Bibliographie}

[1]https://fr.wikiversity.org/wiki/Calcul_avec_les_nombres_complexes

[2]Scénarisation pédagogique dirigée par les intentions, Valérie EMIN (IFÉ-ENSL, Lyon), JeanPhilippe PERNIN (LIG, Grenoble), Viviane GUÉRAUD (LIG, Grenoble), sticef.org V18, 2011

[3]Dispositifs hybrides. Nouvelles perspectives pour une pédagogie renouvelée de l'enseignement supérieur. Rapport final (rapport_final_hysup_12.pdf; Annexes complémentaires: questionnaire_enseignants.pdf, questionnaire_etudiants.pdf)

\section{BIOGRAPHIE DES AUTEURS :}

Jean-Marc Routoure : Jean-Marc Routoure est professeur des Universités à l'université de Caen Normandie. Il s'intéresse aux phénomènes de fluctuations à l'origine du bruit dans les composants électroniques. Responsable d'une mention « sciences pour l'ingénieur » dans une licence, il expérimente dans le domaine des enseignements hybrides présentiel et à distance.

Corentin Jorel : Corentin Jorel est maître de conférence à l'Université de Caen Normandie. Après avoir travaillé sur les détecteurs supraconducteur à très basse température et sur l'optimisation de couches minces high $\mathrm{k}$ pour la microélectronique, ses recherches concernent le développement de capteurs originaux du matériaux au démonstrateur. L'introduction des nouvelles technologies dans l'enseignement l'intéresse particulièrement.

Didier Robbes : Didier Robbes est professeur des Universités à l'UFR des sciences de l'université de Caen Normandie. Sa spécialité première dans le domaine des capteurs de champ magnétique a évolué vers les capteurs bolométriques puis vers les capteurs de champ électrique. Ces spécialités recherche ont également été valorisées dans ses publications à caractères pédagogiques, notamment dans le cadre des colloques CETSIS.

Jeanine Berthier : Jeanine Berthier est ingénieure pédagogique au Centre d'Etude Multimédia Universitaire de l'Université de Caen Normandie. Elle accompagne les enseignants dans leur démarche de pédagogie numérique. Elle est également enseignante dans le master professionnel « Education, formation et mutations sociales : ingénierie et professionnalité » (Module Les TIC pour l'éducation et la formation) et la licence 3 Sciences du langage (module Ingénierie pédagogique).

Anne Garnavault-Remy : Anne Garnavault Remy est chargée de mission au Centre d'Etude Multimédia Universitaire de l'Université de Caen Normandie. Elle a en charge le déploiement et la maintenance du logiciel de pédagogie numérique moodle au sein de l'Université. Elle prend en charge la formation des enseignants à ce logiciel. 


\section{ANNEXE}

\section{Vérification des pré-requis 1 et 2 (PR1 et PR2)}

\subsection{Questions générales}

Chaque propositions devaient être évalués par les étudiants en utilisant le barème suivant : 1 - avis très négatif ou pas du tout d'accord / 2 - avis négatif ou pas d'accord / 3 - avis positif ou d'accord / 4 avis très positif ou très d'accord.

\begin{tabular}{|c|c|c|c|c|}
\hline & 1 & 2 & 3 & 4 \\
\hline L'objectif et les consignes étaient clairement définis et suffisants ? & 0 & 0 & 88 & 13 \\
\hline $\mathrm{PR} 1 / \mathrm{PR} 2$ en $\%$ & 13 & 0 & 75 & 13 \\
\hline Les ressources proposées en auto-apprentissage étaient adaptées aux & 0 & 0 & 88 & 13 \\
\hline objectifs d'apprentissage ? PR1/PR2 en \% & 0 & 25 & 63 & 13 \\
\hline L'évaluation était pertinente et permettait de vérifier ses acquis ? & 0 & 0 & 88 & 13 \\
\hline $\mathrm{PR} 1 / \mathrm{PR} 2$ en $\%$ & 13 & 13 & 75 & 0 \\
\hline
\end{tabular}

1.2. Pouvez-vous préciser le temps consacré à l'apprentissage de cette partie : étude des ressources proposées et réalisation du QCM (estimation).

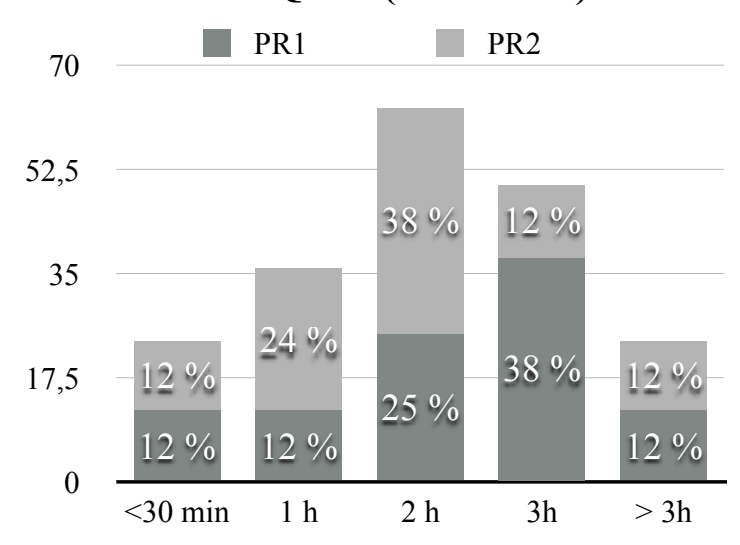

1.3. Pour le QCM, le nombre de tentatives étaient illimité, pouvez-vous préciser le nombre de tentatives que vous avez réalisées

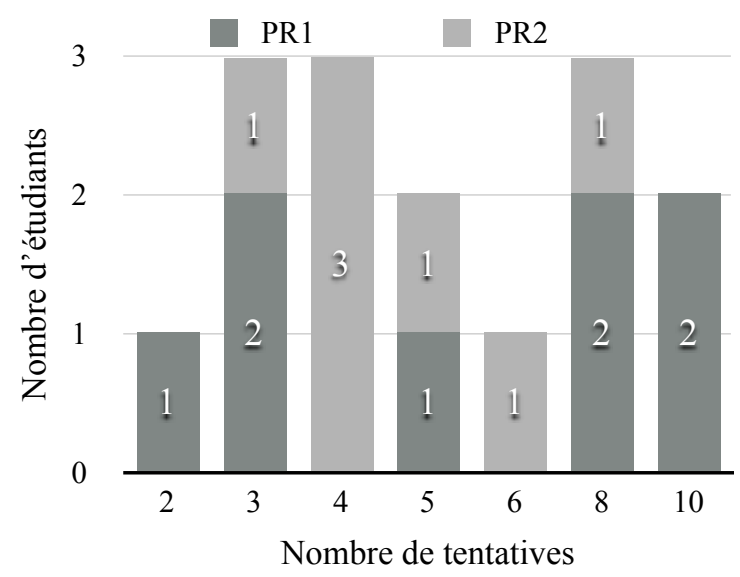


1.4. Avez-vous obtenu la note maximale de 10 ?

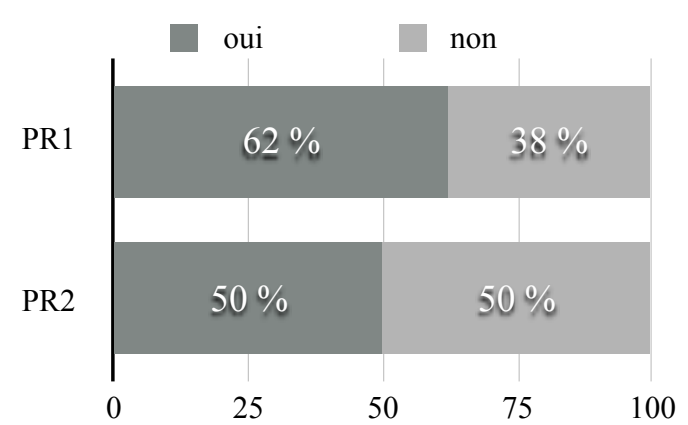

\subsection{Commentaires libres :}

PR1 :

- Pas nécessaire.

- Non

- Pas de remarques supplémentaires

- La partie nombres complexe était plutôt bonne !

- la vérification pré-requis 1 m'a permis d'apprendre et de comprendre à mon rythme .

- pas de remarque particulière

\section{PR2 :}

- Pas nécessaire.

- Non

- Le cours proposé n'était peut être pas assez détaillé pour la mise en pratique.

- Je n'ai pas fait cette partie car je n'ai pas compris le cours mis en ligne.

- Par contre les explications données en cours et en td m'ont permis de bien comprendre ce dont j'avais besoin pour l'assimilation des objectifs du cours

- Idem

- pas très complet et clair

\subsection{Travaux pratiques}

\subsubsection{Questions générales}

Chaque propositions devaient être évaluées par les étudiants en utilisant le barème suivant : 1 - avis très négatif ou pas du tout d'accord / 2 - avis négatif ou pas d'accord / 3 - avis positif ou d'accord / 4 avis très positif ou très d'accord.

\begin{tabular}{l|l|l|l|l} 
& 1 & 2 & 3 & 4 \\
\hline L'objectif et les consignes étaient clairement définis et suffisants ? & 0 & 0 & 75 & 25 \\
L'accès aux ressources, contenus et activités en ligne était adapté à la & 0 & 0 & 63 & 38 \\
réalisation du TP ? & & & & \\
L'espace de cours en ligne était convivial et facile à utiliser. & 0 & 0 & 63 & 38 \\
Vous avez su vous repérer facilement dans la formation ("à quelle étape du & 0 & 0 & 50 & 50 \\
TP suis-je?"). & & & &
\end{tabular}




\subsubsection{Le format des éléments de ce TP a facilité la réalisation du TP?}

\begin{tabular}{|c|c|c|c|c|}
\hline \multicolumn{2}{|l|}{1} & 2 & 3 & 4 \\
\hline \multirow{2}{*}{$\begin{array}{l}\text { Travail préparatoire proposé sous forme de QCM. } \\
\text { Énoncé du TP traité sous forme de MindMap. }\end{array}$} & 0 & 25 & 63 & 13 \\
\hline & 0 & 13 & 63 & 25 \\
\hline Saisie de vos résultats en ligne sur la plateforme. & 0 & 0 & 75 & 25 \\
\hline $\begin{array}{l}\text { Accès conditionnel aux ressources (nécessité d'avoir réalisé l'activité X pour } \\
\text { accéder à l'activité Y). }\end{array}$ & 0 & 25 & 63 & 13 \\
\hline $\begin{array}{c}\text { Les vidéos de pratiques expérimentales ont représenté une véritable valeur } \\
\text { ajoutée. }\end{array}$ & 0 & 0 & 25 & 75 \\
\hline
\end{tabular}

\subsubsection{Avez-vous consulté toutes les vidéos?}

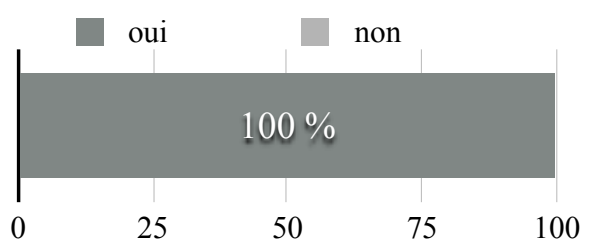

4.2.4.Avez-vous rencontré des difficultés dans la réalisation de ce TP ?

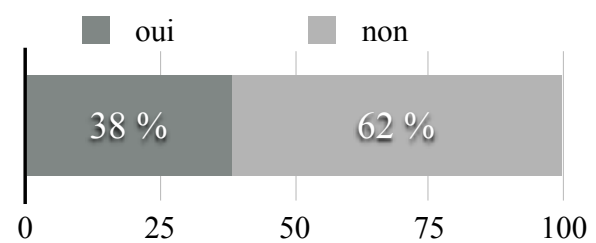

Si oui, lesquelles?

- signal de code en morse

- j'étais tout seul et pas de binôme,donc moins performant

- Je ne peux pas bien écouter codes en morse.

\subsubsection{Commentaires libres sur les TP :}

- Pas nécessaire.

- L'accès conditionnel $n$ a pas très bien marché pour le TP 1 (accès au video)

- Le fait de décoder du morse était motivant.

- Au début on avait tendance à refaire comme sur la vidéo sans comprendre, mais au final non.

- Pour ma part j'ai trouvé que ces TPs étaient très bien organisés, ça m'a permis de m'exercer et d'éclairer certains points du cours (notamment l'interprétation d'un diagramme de bode)

- bien dans l'ensemble

\subsection{Travail de groupe en travaux pratiques : signaux codés en morse}

\subsubsection{Pour vous, les activités collaboratives favorisent-elles :}

\begin{tabular}{c|c:c:c:c} 
& 1 & 2 & 3 & 4 \\
\hline Le développement de compétences techniques. & 0 & 0 & 75 & 25 \\
La motivation. & 0 & 0 & 50 & 50 \\
La confiance en soi. & 0 & 0 & 88 & 12 \\
La connaissance des autres. & 0 & 13 & 75 & 13 \\
La prise de décision. & 0 & 13 & 75 & 13
\end{tabular}




\subsubsection{Commentaires libres sur les activités collaboratives}

- Pas nécessaire.

- Pas de remarques

- Le travail de groupe permet de s'entraider et de ne pas rester bloqué tout seul dans son coin. C'est plus agréable.

- L'intérêt à mon avis réside dans le fait qu'il y'ait un "échange de connaissances" qui permet à chaque membre du groupe d'apprendre de son partenaire, personnellement c'était mon cas.

- Cela a était pour moi très divertissant et pédagogique.

- pas de groupe me concernant

\section{4.modalités d'apprentissage pour ce dispositif de formation en ligne}

\subsubsection{Par rapport à un TP (sans usage de la plateforme et ressources en ligne), diriez-vous que :}

Je suis plus motivé.

Les tâches qui me sont proposées sont plus intéressantes.

Je me suis plus impliqué dans le TP.

Je peux davantage faire le lien avec des activités futures professionnelles.

L'usage des technologies a facilité mon apprentissage.

Le cours m'a amené davantage à réfléchir sur ma manière d'apprendre.

Le cours m'a permis de gérer moi-même mes ressources d'apprentissage.

La qualité de mes productions est améliorée.

La forme de mes productions est améliorée.

Le cours a favorisé l'interaction avec les autres étudiants.

Ce TP a nécessité un temps de travail plus important.

\begin{tabular}{|l|l|l|l}
1 & 2 & 3 & 4 \\
\hline 0 & 0 & 88 & 12 \\
\hdashline 0 & 13 & 75 & 13 \\
\hdashline 0 & 13 & 75 & 13 \\
\hdashline 0 & 50 & 50 & 0 \\
\hdashline 0 & 13 & 75 & 13 \\
\hdashline 0 & 13 & 63 & 25 \\
\hdashline 0 & 0 & 88 & 13 \\
\hdashline 0 & 25 & 50 & 25 \\
\hdashline 0 & 0 & 75 & 25 \\
\hdashline 0 & 13 & 63 & 25 \\
\hdashline 0 & 38 & 38 & 25
\end{tabular}

\subsubsection{Commentaires libres sur les modalités d'apprentissage.}

- Pas nécessaire.

- Pas de remarque

- Pas de remarques supplémentaires.

- La formation en ligne est très agréable car on peut bien organiser notre travail et mieux le comprendre.

- Rien

- plus de TP du type "code morse"

- rien 